\title{
Uma breve comparação de modelos de memórias associativas fuzzy em problemas de guiagem autônoma
}

\section{A brief comparison of fuzzy associative memory models for guiding autonomous problems}

\author{
Guilherme Augusto de Lima Freitas ${ }^{1}$; Marcos Eduardo Valle ${ }^{2}$
}

\section{Resumo}

Memórias associativas fuzzy (FAMs) são modelos inspirados na capacidade do cérebro humano de armazenar e recordar informação. Esses modelos podem ser usados para armazenar associações de conjuntos fuzzy e, portanto, podem ser usados como métodos de inferência em controladores fuzzy. Diversos modelos de FAMs foram desenvolvidos nos últimos anos, mas não temos conhecimento de um trabalho comparando o desempenho dos novos modelos em problemas de controle. Nesse artigo, comparamos brevemente o desempenho de algumas FAMs nos problemas de guiagem autônoma: Backing-up a Truck (BT) e Backing-up a Truck and Trailer (BTT). Em particular, observamos que as memórias associativas fuzzy implicativas duais (co-IFAMs) fornecem uma alternativa atraente aos modelos tradicionais como os de Kosko e Mamdani.

Palavras-chave: Teoria dos conjuntos fuzzy, memória associativa fuzzy, método de inferência, controlador fuzzy.

\begin{abstract}
Fuzzy associative memories (FAMs) are models inspired in the human brain ability to store and recall information. These models can be used for the storage of associations of fuzzy sets and, thus, they can be used as inference engines in fuzzy controllers. Several FAM models have been developed in the last years, but we are not aware of a work comparing the performance of novel FAMs in control. In this paper, we briefly investigate the performance of some FAMs in the automatic guidance problems of backing-up a truck (BT) and backing-up a truck and trailer (BTT). In particular, we note that the dual implicative fuzzy associative memories (co-IFAMs) constitute an interesting alternative to traditional models such as that of Kosko and Mamdani.
\end{abstract}

Key words: Fuzzy set theory, fuzzy associative memory, inference engine, fuzzy controller.

\footnotetext{
Discente de graduação em Engenharia Elétrica, Universidade Estadual de Londrina; gfreitas.eng@gmail.com

2 Docente do Departamento de Matemática da Universidade Estadual de Londrina; valle@uel.br
} 


\section{Introdução}

Sistemas fuzzy, em particular controladores fuzzy, vêm sendo aplicados com sucesso numa grande variedade de problemas, incluindo o controle de metrôs e robôs, além de serem encontrados em produtos como máquinas de lavar-roupas, câmeras de vídeo, carros, entre outros (LEE, 1990a; 1990b; JANTZEN, 2007; IBRAHIM, 2004). Um aspecto positivo dos controladores fuzzy é que esses são geralmente modelos robustos capazes de apresentar um desempenho satisfatório mesmo diante de informações vagas ou imprecisas (KOSKO, 1992; PEDRYCZ; GOMIDE, 2007).

De um modo geral, um controlador fuzzy é composto por um conjunto de regras fuzzy da forma SE-ENTÃO, juntamente com um método de inferência e estratégias de fuzzificação e defuzzificação. As regras fuzzy podem ser estabelecidas por um especialista ou de forma autônoma usando um conjunto representativo de dados (PEDRYCZ; GOMIDE, 2007; PEDRYCZ; SKOWRON; KREINOVICH, 2008). Em muitos casos, as regras substituem um modelo matemático tradicional que descreve a relação entre as entradas e saídas do controlador. Os modelos de Kosko e Mamdani são exemplos de métodos de inferência (PEDRYCZ; GOMIDE, 2007).

As memórias associativas fuzzy (FAMs) também podem ser usadas como método de inferência em controladores fuzzy na qual ambos antecedentes e consequentes são conjuntos fuzzy (KONG; KOSKO, 1992; KOSKO, 1992). De fato, uma FAM é uma aplicação contínua sobre conjuntos fuzzy. Sobretudo, regras fuzzy da forma "SE A ${ }^{\gamma}$, ENTÃO $\mathrm{B}^{\gamma}$ " podem ser vistas como associações $\left(\mathrm{A}^{\gamma}, \mathrm{B}^{\gamma}\right)$ que são memorizadas no modelo. Posteriormente, podese apresentar um conjunto fuzzy A para a FAM que recordará um conjunto fuzzy $\mathrm{B}$, que corresponde à saída do sistema de regras fuzzy. Além disso, uma FAM é também um modelo matemático inspirado na habilidade do cérebro humano de recordar por associação (KOSKO, 1992; VALLE, 2007).
O interesse em FAMs surgiu no início dos anos 1990 com os modelos max-min e max-produto de Kosko (KOSKO, 1992). Apesar desses modelos apresentarembonsresultados em diversos problemas, as duas FAMs de Kosko apresentam uma capacidade absoluta de armazenamento extremamente baixa. De fato, em geral podemos armazenar e recordar com sucesso uma única associação $\left(\mathrm{A}^{\gamma}, \mathrm{B}^{\gamma}\right)$ nesses modelos. Diversos pesquisadores apresentaram modelos que superam essa limitação. Por exemplo, Chung e Lee generalizaram as FAMs de Kosko considerando modelos que calculam o máximo de normas triangulares da lógica fuzzy (CHUNG; LEE, 1996). Sussner e Valle introduziram as memórias associativas fuzzy implicativas (IFAMs), que podem ser vistas como versões melhoradas das FAMs de Chung e Lee para o armazenamento de padrões (SUSSNER; VALLE, 2006a). Eles também introduziram modelos duais que calculam o mínimo de conormas triangulares. Ambas IFAMs e IFAMs duais empregam uma estratégia de armazenamento ótima que, num certo sentido, produz a melhor FAM para um dado conjunto de associações $\left\{\left(\mathrm{A}^{\gamma}, \mathrm{B}^{\gamma}\right)\right.$ : $\gamma=1, \ldots, \mathrm{k}\}$. As IFAMs foram aplicadas com sucesso em problemas de reconstrução de imagens e previsão de séries temporais (SUSSNER; VALLE, 2006a; 2006b; 2007; SUSSNER; MIYASAKI; VALLE, 2009; VALLE, 2007; 2010), entretanto, não temos conhecimento de nenhuma aplicação desses modelos em problemas de controle.

Neste artigo, estaremos interessados na aplicação das FAMs como controladores fuzzy nos problemas de guiagem autônoma Backing up a Truck (BT) e Backing up a Truck- and-Trailer (BTT). Lembrese que o problema BT consiste em estacionar um caminhão num pátio efetuando apenas movimentos para trás. Já o problema BTT consiste, nas mesmas condições do problema BT, em estacionar uma carreta acoplada a um caminhão. Esses são problemas não-lineares de guiagem autônoma de veículo e de veículo articulado que foram amplamente investigados nas últimas décadas (NGUYEN; WIDROW, 1989; TANAKA; KOSAKI, 1997; 
RIID; RÜSTERN, 2001; YANG; YUAN; YU, 2006; WANG; ZHANG, 2009). Em particular, Kong e Kosko apresentaram uma abordagem atraente para o BTT usando FAMs (KONG; KOSKO, 1992; KOSKO, 1992). Nesse artigo comparamos o desempenho das FAMs de Kosko, o modelo de Mamdani, as IFAMs e as IFAMs duais em ambos problemas BT e BTT.

O artigo está organizado como segue. Na próxima seção apresentaremos uma breve revisão das principais FAMs, incluindo equações que resultam da aplicação desses modelos em problemas de controle. Na seção 3, apresentamos uma abordagem fuzzy para os problemas de guiagem autônoma BT e BTT, bem como o resultado de simulações computacio nais usando diversas FAMs. O artigo termina com a conclusão na seção 4 .

\section{Conceitos Básicos de FAMs}

Uma FAM corresponde à uma aplicação contínua entre conjuntos fuzzy. Antes de apresentarmos as FAMs que serão consideradas neste artigo, gostaríamos de revisar alguns conceitos da teoria dos conjuntos fuzzy.

A teoria dos conjuntos fuzzy foi introduzida por Zadeh no intuito de modelar conceitos vagos ou incertos como as noções de "pessoa jovem" e "ângulo pequeno" (KLIR; YUAN, 1995; PEDRYCZ; GOMIDE, 2007). Matematicamente, um conjunto fuzzy corresponde a uma função A : U $\rightarrow[0,1]$, onde $U$ é o universo na qual o conjunto está definido. $\mathrm{O}$ valor $\mathrm{A}(\mathrm{u})$ representa o grau de pertinência do elemento u $\epsilon$ U no conjunto fuzzy $A$. A classe de todos os conjuntos fuzzy definidos em $U$ é denotada por $F(U)$. Dado u $\in U$, denotaremos por $I_{u} \in F(U)$ o conjunto indicador definido como segue para todo $\mu \in U$ :

$$
I_{u}(\mu)=\left\{\begin{array}{l}
1, \text { se } \mu=u, \\
0, \text { caso contrário. }
\end{array}\right.
$$

Um conjunto fuzzy $\mathrm{R}$ definido no produto cartesiano $U \times V$, i.e., $R \in F(U \times V)$, é chamado relação fuzzy.

De agora em diante, suponha que temos um conjunto de associações $\left\{\left(\mathrm{A}^{\gamma}, \mathrm{B}^{\gamma}\right): \gamma=1, \ldots, \mathrm{k}\right\}$, onde $\mathrm{A}^{\gamma} \in \mathrm{F}(\mathrm{U})$ e $\mathrm{B}^{\gamma} \in \mathrm{F}(\mathrm{V})$ são conjuntos fuzzy definidos em $\mathrm{U}$ e $\mathrm{V}$, respectivamente. Lembre-se que cada associação pode ser vista como uma regra da forma: "SE $\mathrm{A}^{\gamma}$, ENTÃO $\mathrm{B}^{\gamma}$ ”. Neste artigo, assumimos que as regras são conhecidas a priori.

Uma FAM corresponde à uma aplicação $\mathrm{M}: \mathrm{F}$ $(\mathrm{U}) \rightarrow \mathrm{F}(\mathrm{V})$. Deseja-se que essa aplicação satisfaça $\mathrm{M}\left(\mathrm{A}^{\gamma}\right)=\mathrm{B}^{\gamma}$ para todo $\gamma=1, \ldots, \mathrm{k}$. Dizemos que a FAM armazenou corretamente as associações se essa equação for válida para todo $\gamma$ (VALLE, 2007). Deseja-se também que a FAM represente uma aplicação contínua (KOSKO, 1992). Isso significa que, se A é um conjunto fuzzy próximo de $A^{\gamma}$, então a saída $M(A)$ também é próxima de

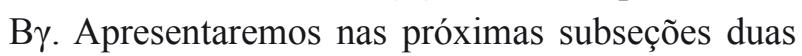
classes importantes de FAMs. Antes, porém, gostaríamos de esclarecer um pouco mais a noção de continuidade de uma FAM, que depende do conceito de proximidade entre conjuntos fuzzy.

A noção de proximidade entre conjuntos fuzzy pode ser expressa utilizando uma medida de similaridade S (PEDRYCZ; GOMIDE, 2007; DUBOIS; PRADE, 1980). Um exemplo de medida de similaridade é a razão entre a cardinalidade da interseção pela cardinalidade da união de dois conjuntos fuzzy. Matematicamente, a equação

$$
\mathrm{S}\left(\mathrm{A}, \mathrm{A}^{\gamma}\right)=\frac{\operatorname{Card}\left(\mathrm{A \cap A}^{\gamma}\right)}{\operatorname{Card}\left(\mathrm{AUA}^{\gamma}\right)}
$$

fornece uma medida para a similaridade entre os conjun- tos fuzzy A e $\mathrm{A}^{\gamma}$. O grau de veracidade da afirmação "A $\subseteq \mathrm{A}^{\gamma}$ e $\mathrm{A}^{\gamma} \underline{\mathrm{C}} \mathrm{A}$ " é outro exemplo de medida de similaridade. De fato, existe uma vasta literatura sobre medidas de similaridade, mas não entraremos em detalhes sobre esse assunto nesse artigo (PAPPIS; KARACAPILIDIS, 1993; CHEN; YEH; HSIAO, 1995). Finalmente, a continuidade 
de uma FAM M pode ser avaliada no sentido proposto por Perfilieva e Lehmke para sistemas de regras fuzzy (PERFILIEVA; LEHMKE, 2006). Especificamente, dizemos que $\mathrm{M}: \mathrm{F}(\mathrm{U}) \rightarrow \mathrm{F}(\mathrm{V})$ é contínua no sentido de Perfilieva-Lehmke sobre o conjunto de associações $\left\{\left(\mathrm{A}^{\gamma}, \mathrm{B}^{\gamma}\right): \gamma=1, \ldots, \mathrm{k}\right\}$ se a inequação

$$
\mathrm{S}\left(\mathrm{M}(\mathrm{A}), \mathrm{B}^{\gamma}\right) \geq \mathrm{S}\left(\mathrm{A}, \mathrm{A}^{\gamma}\right),
$$

vale para todo $\gamma=1, \ldots, \mathrm{k}$ e para qualquer conjunto fuzzy A $\in \mathrm{F}$ (U) (OLIVEIRA, 2010). Em termos gerais, (3) diz que o padrão recordado $\mathrm{M}(\mathrm{A})$ é pelo menos tão similar a $\mathrm{B}^{\gamma}$ quanto $\mathrm{A}$ é similar a $\mathrm{A}^{\gamma}$.

\section{A Classe das FAMs Sup- $t$}

Muitas FAMs são descritas pela equação

$$
\mathrm{M}(\mathrm{A})(\mathrm{v})=\sup _{\mathrm{u} \in \mathrm{U}}\{\mathrm{R}(\mathrm{u}, \mathrm{v}) \mathrm{t} \mathrm{A}(\mathrm{u})\},
$$

onde $\mathrm{R} \in \mathrm{F}(\mathrm{U} \times \mathrm{V})$ é uma relação fuzzy e $\mathrm{t}$ : [0, $1] \times[0,1] \rightarrow[0,1]$ denota uma norma triangular ou, simplesmente, t-norma (PEDRYCZ; GOMIDE, 2007). Lembre-se que uma t-norma é uma operação comutativa, associativa, monótona crescente que satisfaz a t $1=$ a e a t $0=0$ para todo a $€[0,1]$. As seguintes operações são exemplos de t-norma:

Mínimo: $\quad \mathrm{a} \mathrm{t}_{\mathrm{m}} \mathrm{b}=\min (\mathrm{a}, \mathrm{b})$,

Lukasiewicz: $\quad$ a $\mathrm{t}_{1} \mathrm{~b}=\max (\mathrm{a}+\mathrm{b}-1,0)$.

Um modelo descrito por (4) é chamado FAM Sup-t. As FAMs generalizadas de Chung e Lee são exemplos de FAMs Sup-t (CHUNG; LEE, 1996). As IFAMs são também exem- plos de FAMs Sup-t (VALLE, 2007). A diferença entre esses modelos está na forma como a relação fuzzy $\mathrm{R} \in \mathrm{F}(\mathrm{U} \times \mathrm{V})$ é determinada.

Dado um conjunto de associações $\left\{\left(\mathrm{A}^{\gamma}, \mathrm{B}^{\gamma}\right): \gamma\right.$ $=1, \ldots, \mathrm{k}\}$, a relação fuzzy é definida como segue nas FAMs generalizadas de Chung e Lee:

$$
\check{\mathrm{R}}(\mathrm{u}, \mathrm{v})=\max _{\gamma=1: \mathrm{k}}\left\{\mathrm{A}^{\gamma}(\mathrm{u}) \mathrm{t} \mathrm{B}^{\gamma}(\mathrm{v})\right\} \text {. }
$$

Por outro lado, nas IFAMs, a relação fuzzy é definida através da equação

$\hat{\mathrm{R}}(\mathrm{u}, \mathrm{v})=\underset{\gamma=1: \mathrm{k}}{\min }\left\{\mathrm{A}^{\gamma}(\mathrm{u}) \rightarrow \mathrm{B}^{\gamma}(\mathrm{v})\right\}$,

onde $\rightarrow$ denota a implicação residual associada a t-norma usada em (4). Por exemplo, a implicação residual associada à t-norma de Lukasiewicz é dada pela seguinte equação para todo $a, b \in[0,1]$ (PEDRYCZ; GOMIDE, 2007):

Lukasiewicz: $\quad \mathrm{a} \rightarrow{ }_{1} \mathrm{~b}=\min (1,1-\mathrm{a}+\mathrm{b})$.

É importante observar que a relação fuzzy dada por (8) é ótima no seguinte sentido: Se existe uma FAM Sup-t capaz de armazenar todas as associações $\left(\mathrm{A}^{\gamma}\right.$, $\mathrm{B}^{\gamma}$ ), então a FAM dada por (4), considerando a relação dada por (8), também consegue armazenar todas as associações (VALLE, 2007). Infelizmente, não existe um resultado similar para as FAMs dadas por (4) considerando a relação dada por (7).

\section{A Classe das IFAMs Duais}

De um modo similar a equação (4), podemos definir FAMs como

$$
\mathrm{W}(\mathrm{A})(\mathrm{v})=\inf _{\mu \in \mathrm{U}}\{\mathrm{S}(\mu, \mathrm{v}) \mathrm{s} \mathrm{A}(\mu)\},
$$

onde $\mathrm{S} \in \mathrm{F}(\mathrm{U} \times \mathrm{V})$ é uma relação fuzzy e $\quad s:[0,1] \times[0,1] \rightarrow[0,1]$ representa uma conorma triangular ou, simplesmente, t-conorma (PEDRYCZ; GOMIDE, 2007). Lembre-se que uma t-conorma é uma operação comutativa, associativa, monótona crescente que satisfaz a s $1=1$ e a s $0=$ a para todo a $€[0,1]$. As seguintes operações são exemplos de t-conorma:

$$
\begin{array}{ll}
\text { Máximo: } & \text { a s }{ }_{m} b=\max (a, b), \\
\text { Lukasiewicz: } & \text { a s }_{1} b=\min (a+b, 1) .
\end{array}
$$

Dado um conjunto de associações $\left\{\left(\mathrm{A}^{\gamma}, \mathrm{B}^{\gamma}\right): \gamma=\right.$ $1, \ldots, \mathrm{k}\}$, a relação fuzzy em (10) pode ser obtida através da equação:

$\check{\mathrm{S}}(\mathrm{u}, \mathrm{v})=\max _{\gamma=1: \mathrm{k}}\left\{\mathrm{A}^{\gamma}(\mathrm{u})<\neq \mathrm{B}^{\gamma}(\mathrm{v})\right\}$. 
A operação $\longleftarrow$ denota a co-implicação, também chamada implicação dual, residual associada a t-conorma usada em (10) (DE BAETS, 1997; SUSSNER; VALLE, 2006a). A expressão $\mathrm{a} \leftarrow_{\mathrm{s}}$ b pode ser interpretada como "a não é necessário para b". As seguintes equações representam as coimplicações associadas às t-conormas dadas em (11) e (12), respectiva mente:

Gödel: $a<\neq_{m} b=b \cdot I(a<b)$

Lukasiewicz: $a<\neq, b=\max (b-a, 0)$.

Em (14), a expressão I $(\mathrm{a}<\mathrm{b})$ é definida como

$\mathrm{I}(\mathrm{a}<\mathrm{b})=\left\{\begin{array}{l}1, \text { se } \mathrm{a}<\mathrm{b}, \\ 0, \text { caso contrário. }\end{array}\right.$

Os modelos dados por (10) e (13) são chamados IFAMs duais, pois podem ser obtidos através de (4) e (8) usando conceitos de dualidade como negação e adjunção (SUSSNER; VALLE, 2006a). Devido a co-implicação usada em (13), as IFAMs duais também serão referidas como co-IFAMs.

\section{Aplicação de FAMs em Problemas de Guiagem Autônoma}

Modelos de memórias associativas fuzzy foram aplicados com sucesso em diversos tipos de problemas, incluindo reconhecimento e reconstrução de imagens (SUSSNER; VALLE, 2006a; VALLE; SUSSNER, 2008; VALLE, 2009; 2010) e previsão de séries temporais (SUSSNER; VALLE, 2006b; 2007; SUSSNER; MIYASAKI; VALLE, 2009). Neste trabalho estamos particularmente interessados na aplicação de FAMs em problemas de guiagem autônoma, na qual foram obtidos resultados bastante satisfatórios (KONG; KOSKO, 1992; KOSKO, 1992; TANAKA; KOSAKI, 1997; IBRAHIM, 2004; KOGA; HORIO; YAMAKAWA, 2006). Precisamente, nas subseções seguintes apresentamos uma comparação de FAMs nos problemas "Backing up a Truck" e "Backing up a Truck and Trailer" (BTT).

\section{O Problema "Backing up a Truck"}

O problema BT consiste em estacionar um caminhão (veículo) num determinado local de um pátio efetuando apenas movimentos para trás (marcha-ré) e parando com um ângulo de $90^{\circ}$ entre o caminhão e o eixo (horizontal) do estacionamento (KONG; KOSKO, 1992; KOSKO, 1992). A solução desse problema é obtida determinando o ângulo $\theta$ que as rodas formam com o eixo principal do veículo quando esse se encontra na posição $(\mathrm{x}, \mathrm{y})$ e forma um ângulo $\varphi$ com respeito ao eixo horizontal. Precisamente, conhecidos a posição $(\mathrm{x}, \mathrm{y})$ e os ângulos $\varphi$ e $\theta$, a nova posição (x', y') e o novo ângulo $\varphi^{\prime}$ com respeito a horizontal são determinados utilizando as seguintes equações onde d é a distância percorrida pelo caminhão a cada intervalo de tempo (iteração):

$$
\begin{aligned}
& \varphi^{\prime}=\varphi+\theta, \\
& x^{\prime}=x+d \cos \left(\varphi^{\prime}\right), \\
& y^{\prime}=y+d \operatorname{sen}\left(\varphi^{\prime}\right),
\end{aligned}
$$

O modelo do caminhão é mostrado na figura 1.

Figura 1. Modelo do Caminhão.

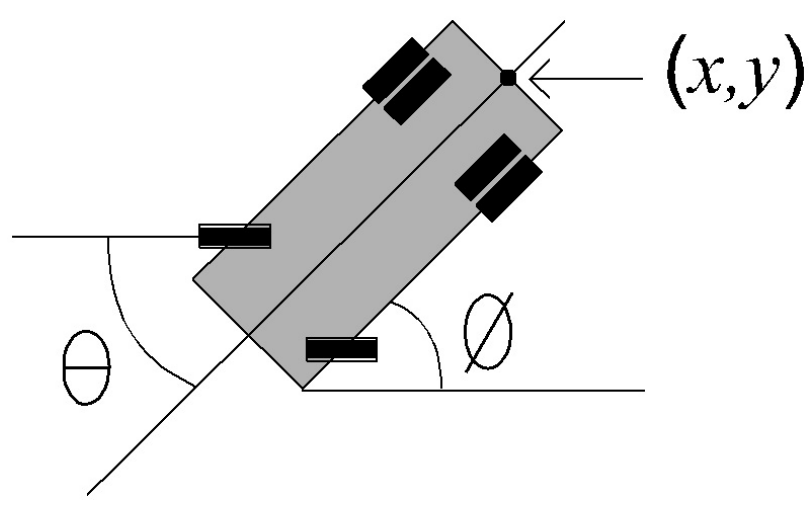

Nesse trabalho, para simplificar a modelagem matemática, supomos que existe uma área suficiente entre o veículo e a vaga de modo que podemos desprezar a coordenada y. 
Assim, as variáveis de estado são x e $\varphi$. A saída do controlador é $\theta$, que deve estar contido num intervalo fechado que repre- senta a amplitude de giro das rodas do veículo. Conforme os trabalhos de Kong e Kosko (KONG; KOSKO, 1992; OSKO, 1992), nesse artigo supomos que o estacionamento corresponde ao plano $[0,100] \times[0,100]$, o intervalo $\left[-30^{\circ}, 30^{\circ}\right]$ é usado para modelar a amplitude de giro das rodas do veículo e a posição final $\left(\mathrm{x}_{\mathrm{f}}, \mathrm{y}_{\mathrm{f}}\right)$ é (50, 100). Supomos também que o veículo se desloca $\mathrm{d}=$ $1 \mathrm{~m}$ a cada intervalo de tempo. É importante observar que, embora a coordenada y não seja considerada no modelo matemático, ela é necessária durante as simulações para determinarmos a posição exata do veículo no interior do pátio.

A figura 2 apresenta as funções de pertinência dos conjuntos fuzzy definidos para as variáveis de entrada e saída do sistema. O controlador fuzzy foi baseado nas regras de inferência da tabela 1, que foram propostas por Kong e Kosko (KONG; KOSKO, 1992; KOSKO, 1992). As funções de pertinência também foram definidas segundo esses autores.

Figura 2. Funções de pertinência das variáveis $\varphi$ e $\varphi_{t}$, x e $\theta$.

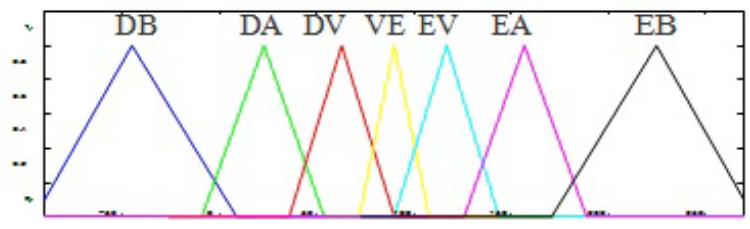

a) Funções de pertinência da variável $\varphi$ e $\varphi_{t}$.

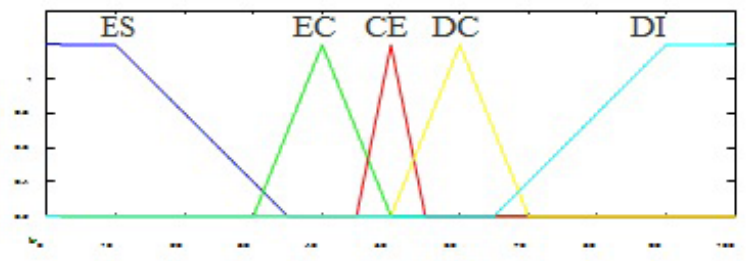

b) Funções de pertinência da variável x.

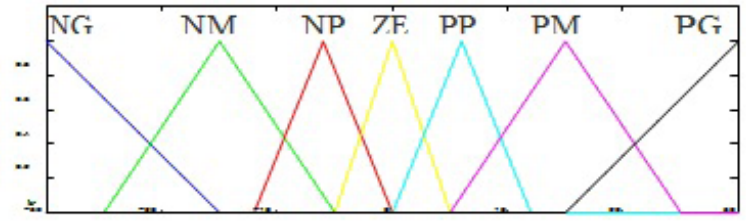

c) Funções de pertinência da variável $\theta$.
Tabela 1 - Regras de inferência do controlador fuzzy para manobrar o caminhão.

\begin{tabular}{cllllll}
\hline & & & & $\mathrm{x}$ & & \\
\hline & & ES & EC & C E & DC & DI \\
\hline DB & PP & PM & PM & PG & PG \\
DA & NP & PP & PM & PG & PG \\
& DV & NM & NP & PP & PM & PG \\
& V E & NM & NM & ZE & PM & PM \\
& EV & NG & NM & NP & PP & PM \\
& EA & NG & NG & NM & NP & PP \\
& EB & NG & NG & NM & NM & NP \\
\hline
\end{tabular}

A tabela 2 mostra os erros de estacionamento baseados nas equações (20) e (21), que apresentam os erros da posição final $(\mathrm{x}, \mathrm{y})$ e do ângulo $\varphi$, respectivamente. Também são mostradas as distâncias percorridas por cada modelo.

$$
\begin{aligned}
& \text { Erro }_{\mathrm{x}, \mathrm{y}}=\sqrt{\left(\mathrm{x}_{\mathrm{f}}-50\right)^{2}+\left(\mathrm{y}_{\mathrm{f}}-100\right)^{2}} \\
& \text { Erro }_{\varphi}=\left|90-\varphi_{\mathrm{f}}\right| .
\end{aligned}
$$

A figura 3 mostra algumas das trajetórias obtidas pelos controladores fuzzy. O modelo de Mamdani apresentou trajetórias muito similares às produzidas pela FAM de Kosko. Assim como a co-IFAM de Lukasiewicz gerou trajetórias similares às produzidas pela co-IFAM de Gödel. Note que as co-IFAMs apresentaram um desempenho simiar aos controladores tradicionais de Mamdani e Kosko, embora não observamos um padrão bem definido em relação aos erros produzidos por cada modelo. Uma análise sistemática do desempenho dos controladores fuzzy será efetuada na próxima subseção considerando um problema mais complexo. 
Tabela 2 - Distância percorrida e erros de estacionamento produzidos pelos controladores fuzzy para o problema "Backing up a Truck".

\begin{tabular}{lccc}
\hline & Erro $_{\mathbf{x}, \mathbf{y}}$ & Erro $_{\boldsymbol{\varphi}}$ & Dist \\
\hline Met. de Mamdani & $0.94 \mathrm{~m}$ & $0.00^{\circ}$ & $76 \mathrm{~m}$ \\
FAM de Kosko & $0.83 \mathrm{~m}$ & $0.00^{\circ}$ & $76 \mathrm{~m}$ \\
IFAM Lukasiewicz & $3.00 \mathrm{~m}$ & $43.87^{\circ}$ & $118 \mathrm{~m}$ \\
co-IFAM de Luk. & $0.87 \mathrm{~m}$ & $2.01^{\circ}$ & $76 \mathrm{~m}$ \\
co-IFAM de Gödel & $0.80 \mathrm{~m}$ & $0.79^{\circ}$ & $76 \mathrm{~m}$ \\
\hline
\end{tabular}

a) Posição inicial: $\left(30,40,-60^{\circ}\right)$.

\begin{tabular}{lccr}
\hline & Erro $_{\mathbf{x}, \mathbf{y}}$ & Erro $_{\boldsymbol{\varphi}}$ & Dist \\
\hline Met. de Mamdani & $0.91 \mathrm{~m}$ & $0.00^{\circ}$ & $95 \mathrm{~m}$ \\
FAM de Kosko & $0.95 \mathrm{~m}$ & $0.00^{\circ}$ & $95 \mathrm{~m}$ \\
IFAM Lukasiewicz & $3.30 \mathrm{~m}$ & $21.76^{\circ}$ & $135 \mathrm{~m}$ \\
co-IFAM de Luk. & $0.91 \mathrm{~m}$ & $1.76^{\circ}$ & $93 \mathrm{~m}$ \\
co-IFAM de Gödel & $0.49 \mathrm{~m}$ & $0.86^{\circ}$ & $93 \mathrm{~m}$ \\
\hline
\end{tabular}

c) Posição inicial: $\left(70,20,-40^{\circ}\right)$.

\begin{tabular}{llcl}
\hline & Erro $_{\mathbf{x}, \mathbf{y}}$ & Erro $_{\boldsymbol{\varphi}}$ & Dist \\
\hline Met. de Mamdani & $0.73 \mathrm{~m}$ & $0.12^{\circ}$ & $67 \mathrm{~m}$ \\
FAM de Kosko & $0.87 \mathrm{~m}$ & $0.02^{\circ}$ & $67 \mathrm{~m}$ \\
IFAM Lukasiewicz & $68.53 \mathrm{~m}$ & $33.35^{\circ}$ & $30 \mathrm{~m}$ \\
co-IFAM de Luk. & $0.78 \mathrm{~m}$ & $1.02^{\circ}$ & $65 \mathrm{~m}$ \\
co-IFAM de Gödel & $0.92 \mathrm{~m}$ & $0.90^{\circ}$ & $66 \mathrm{~m}$ \\
\hline
\end{tabular}

e) Posição inicial: $\left(25,55,240^{\circ}\right)$.

\section{O Problema "Backing up a Truck-and-} Trailer"

O problema BTT consiste em estacionar um caminhão acoplado a uma carreta (veículo articulado) nas mesmas condições do problema BT (KONG; KOSKO, 1992; KOSKO, 1992; TANAKA; KOSAKI, 1997; KOGA; HORIO; YAMAKAWA, 2006). A solução deste problema

\begin{tabular}{lccr}
\hline & Erro $_{\mathbf{x}, \mathbf{y}}$ & Erro $_{\boldsymbol{\varphi}}$ & Dist \\
\hline Met. de Mamdani & $0.50 \mathrm{~m}$ & $0.00^{\circ}$ & $79 \mathrm{~m}$ \\
FAM de Kosko & $0.53 \mathrm{~m}$ & $0.00^{\circ}$ & $79 \mathrm{~m}$ \\
IFAM Lukasiewicz & $7.42 \mathrm{~m}$ & $12.03^{\circ}$ & $110 \mathrm{~m}$ \\
co-IFAM de Luk. & $0.34 \mathrm{~m}$ & $2.13^{\circ}$ & $78 \mathrm{~m}$ \\
co-IFAM de Gödel & $0.21 \mathrm{~m}$ & $0.56^{\circ}$ & $78 \mathrm{~m}$ \\
\hline
\end{tabular}

b) Posição inicial: $\left(35,30,200^{\circ}\right)$.

\begin{tabular}{lccc}
\hline & Erro $_{\mathbf{x}, \mathbf{y}}$ & Erro $_{\boldsymbol{\varphi}}$ & Dist \\
\hline Met. de Mamdani & $0.85 \mathrm{~m}$ & $0.00^{\circ}$ & $95 \mathrm{~m}$ \\
FAM de Kosko & $0.94 \mathrm{~m}$ & $0.00^{\circ}$ & $95 \mathrm{~m}$ \\
IFAM Lukasiewicz & $6.23 \mathrm{~m}$ & $15.40^{\circ}$ & $96 \mathrm{~m}$ \\
co-IFAM de Luk. & $0.16 \mathrm{~m}$ & $1.88^{\circ}$ & $94 \mathrm{~m}$ \\
co-IFAM de Gödel & $0.31 \mathrm{~m}$ & $0.87^{\circ}$ & $94 \mathrm{~m}$ \\
\hline
\end{tabular}

d) Posição inicial: $\left(80,25,30^{\circ}\right)$.

\begin{tabular}{lccc}
\hline & Erro $_{\mathbf{x}, \mathbf{y}}$ & Erro $_{\varphi}$ & Dist \\
\hline Met. de Mamdani & $0.14 \mathrm{~m}$ & $0.00^{\circ}$ & $69 \mathrm{~m}$ \\
FAM de Kosko & $0.16 \mathrm{~m}$ & $0.00^{\circ}$ & $69 \mathrm{~m}$ \\
IFAM Lukasiewicz & $7.05 \mathrm{~m}$ & $29.78^{\circ}$ & $81 \mathrm{~m}$ \\
co-IFAM de Luk. & $0.90 \mathrm{~m}$ & $1.97^{\circ}$ & $69 \mathrm{~m}$ \\
co-IFAM de Gödel & $0.41 \mathrm{~m}$ & $0.99^{\circ}$ & $69 \mathrm{~m}$ \\
\hline
\end{tabular}

f) Posição inicial: $\left(70,40,25^{\circ}\right)$.

também se dá determinando o ângulo $\theta$ das rodas do veículo, mas neste caso devemos considerar o tamanho do veículo (caminhão+carreta). Em outras palavras, o comprimento da cabine 1 e da carreta $\mathrm{L}$ interferem diretamente na trajetória produzida pelo controlador fuzzy. O modelo do veículo articulado é mostrado na figura 4 . Nesse trabalho adotamos $1=3 \mathrm{~m}$ e $\mathrm{L}=5 \mathrm{~m}$. 
Figura 3. Trajetórias produzidas pelos controladores fuzzy baseados em FAMs. O símbolo “ $\square$ ” (azul) representa a trajetória do caminhão.
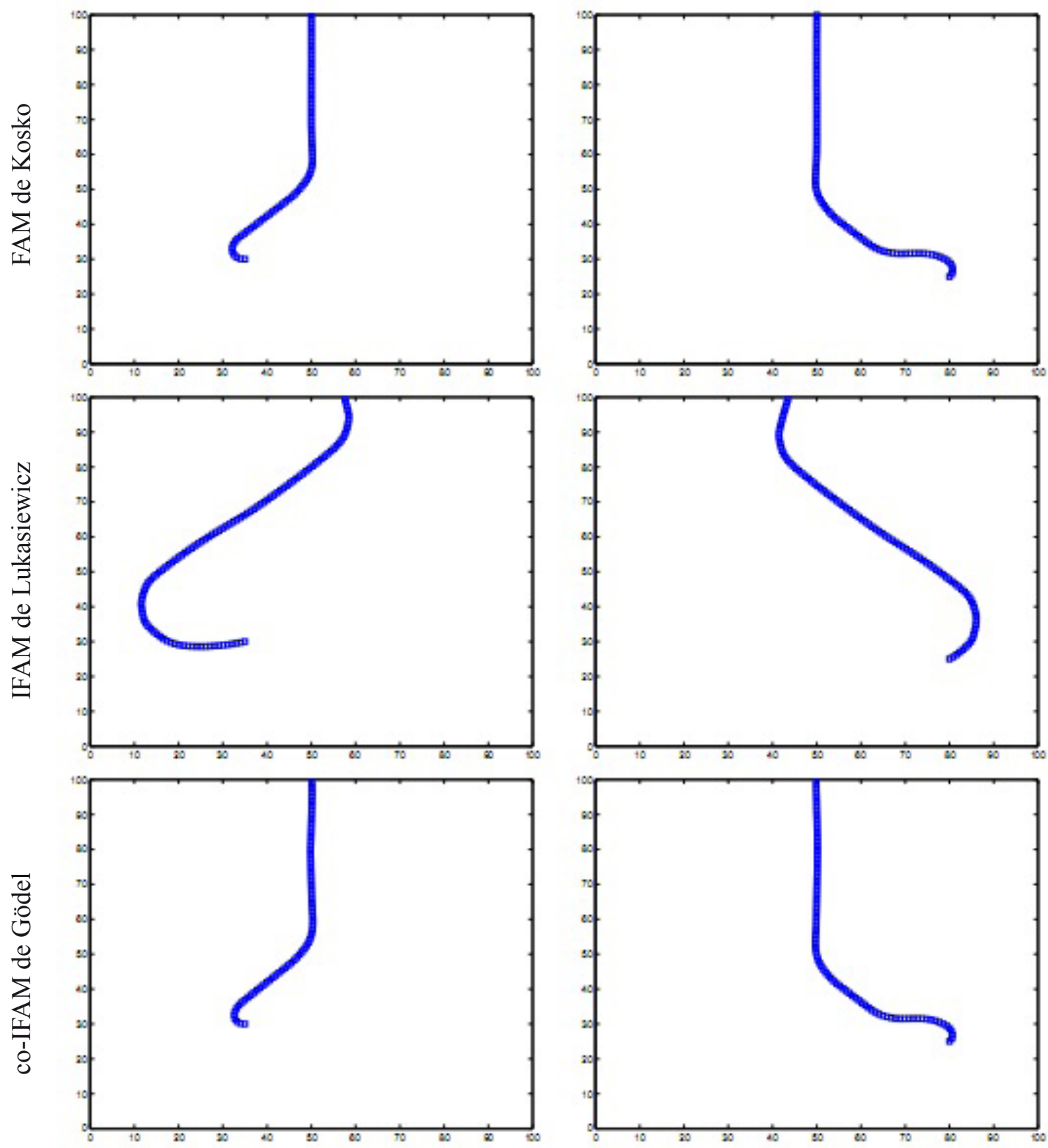

a) Posição inicial: $\left(35,30,200^{\circ}\right)$

b) Posição inicial: $\left(80,25,30^{\circ}\right)$

O ângulo das rodas $\theta$, o ângulo entre a carreta e a horizontal $\varphi \mathrm{t}$ e a coordenada $\mathrm{x}$ possuem os mesmos conjuntos fuzzy do problema BT, cujas funções de pertinência podem ser visualizados na figura 2 . O porque há mais uma variável de estado que deve ser considerada: o ângulo entre a cabine e a carreta $\varphi c$. As funções de pertinência dos conjuntos fuzzy dessa variável são mostradas na figura 5 . 
Figura 4. Modelo da Carreta.

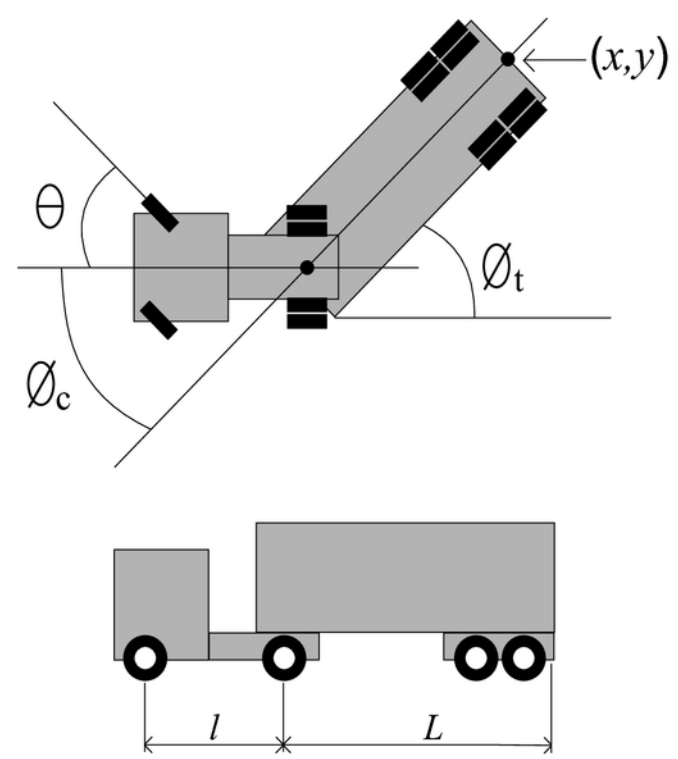

Figura 5. Funções de pertinência da variável $\varphi c$

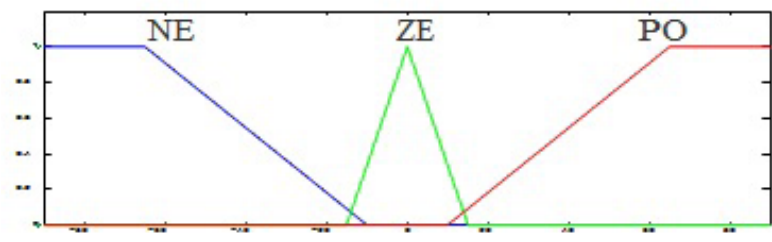

Concluindo, temos um sistema que recebe três variáveis de entrada: $x, \varphi t$ e $\varphi c$ e produz como saída o ângulo $\theta$. O cálculo de $\theta$ é efetuado considerando o conjunto de regras de inferência da tabela 4. O controlador fuzzy que manobra a carreta apresentado nesse artigo constitui uma variação do modelo proposto por Kong e Kosko em (KONG; KOSKO, 1992; KOSKO, 1992). De fato, no nosso modelo utilizamos o conjunto de regras fuzzy para determinar diretamente o ângulo $\theta$. No trabalho de Kosko e Kong, entretanto, o sistema de regras fornece um ângulo $\beta$, que é usado posteriormente para determinar - usado relações trigonométricas - o ângulo $\theta$ das rodas do veículo.

Conhecidos a posição (x, y) e os ângulos $\varphi t, \varphi c$ e $\theta$, podemos utilizar as seguintes equações para determinar a posição e os ângulos após um intervalo de tempo:

$$
\begin{aligned}
& \varphi_{\mathrm{c}}{ }^{\prime}=\varphi_{\mathrm{c}}(1-\mathrm{d} / \mathrm{L})+(\mathrm{d} / \mathrm{l}) \theta, \\
& \varphi_{\mathrm{c}, \mathrm{h}}=\varphi_{\mathrm{c}}+\varphi_{\mathrm{t}}, \\
& \varphi_{\mathrm{c}, \mathrm{h}}{ }^{\prime}=\varphi_{\mathrm{c}, \mathrm{h}}+\varphi_{\mathrm{t}}, \\
& \varphi^{\prime}=\varphi \mathrm{c}, \mathrm{h}^{\prime}-\varphi \mathrm{c}^{\prime}, \\
& \mathrm{x}^{\prime}=\mathrm{x}+\mathrm{d} \cos \left(\varphi^{\prime}\right), \\
& \mathrm{y}^{\prime}=\mathrm{y}+\mathrm{d} \operatorname{sen}\left(\varphi^{\prime}\right),
\end{aligned}
$$

onde $\mathrm{d}=1 \mathrm{~m}$ representa o deslocamento do veículo após o intervalo de tempo (iteração). Note que (23) e (24) apresentam as variáveis $\varphi_{\mathrm{c}, \mathrm{h}}$ e $\varphi_{\mathrm{c}, \mathrm{h}}^{\prime}$ que correspondem ao ângulo entre a cabine e o eixo horizontal. A variável $\varphi_{c, h}^{\prime}$ é usada em (25) para determinar o ângulo entre a carreta e o eixo horizontal. Gostaríamos de observar que (22)(27) foram deduzidas tomando como referência os trabalhos de Tanaka e Kosaki (TANAKA; KOSAKI, 1997) e Koga (KOGA; HORIO; YAMAKAWA, 2006).

A figura 6 mostra algumas trajetórias fornecidas pelos controladores baseados nas FAMs descritas anteriormente para duas posições iniciais $\left(\mathrm{x}, \mathrm{y}, \varphi_{\mathrm{t}}, \varphi_{\mathrm{c}}\right)$. A tabela 3 apresenta os erros da posição final e ângulo de estacionamento, denotados respectivamente por Erro $_{\mathrm{x}, \mathrm{y}}$ e Erro $_{\varphi t}$, e a distância percorrida pelo veículo considerando diversas posições iniciais $\left(\mathrm{x}, \mathrm{y}, \varphi_{\mathrm{t}}, \varphi_{\mathrm{c}}\right)$.

O modelo de Mamdani apresentou trajetórias muito similares às produzidas pela FAM de Kosko. Assim como a co-IFAM de Lukasiewicz gerou trajetórias similares às produzidas pela co-IFAM de Gödel. Note que o controlador baseado na IFAM de Lukasiewicz apresentou resultados pouco satisfatórios. Em vista disso, esse modelo será desconsiderado na análise que segue. Observe também que o maior erro Erro $_{\varphi t}$, que foi produzido pela co-IFAM de Lukasiewicz para a posição inicial do item b), foi de $2.16^{\circ}$. Acreditamos, porém, que esse valor é muito pequeno comparado com as dimensões do problema. Dessa forma, não consideremos a variável Erro ${ }_{\varphi t}$ na análise do desempenho dos controladores fuzzy. 
Para comparar o desempenho dos controladores fuzzy aplicados no problema BTT, consideramos a distância percorrida e o erro da posição final $(\mathrm{x}, \mathrm{y})$. Dessa forma, temos duas variáveis, que podem ser conflitantes no sentido de que um modelo pode percorrer uma distância pequena, mas produzir um Erro $_{x, y}$ grande. Temos, portanto, um problema multi - objetivo e diremos que um controlador produziu um desempenho ótimo no sentido da teoria da otimalidade de Pareto (STADLER, 1988; COELLO; LAMONT; VELDHUIZEN, 2007). Especificamente, diremos que um controlador fuzzy teve um desempenho Pareto-ótimo se nenhum outro controlador produziu ambos distância percorrida e Erro $_{x, y}$ menores.

Tabela 3 - Distância percorrida e erros de estacionamento produzidos pelos controladores fuzzy no problema "Backing up a Truck-and-Trailer".

\begin{tabular}{lccc}
\hline & Erro $_{\mathrm{x}, \mathrm{y}}$ & Erro $_{\varphi \mathrm{t}}$ & Dist \\
\hline Met. de Mamdani & $0.11 \mathrm{~m}$ & $0.15^{\circ}$ & $106 \mathrm{~m}$ \\
FAM de Kosko & $0.72 \mathrm{~m}$ & $0.00^{\circ}$ & $106 \mathrm{~m}$ \\
IFAM Lukasiewicz & $4.53 \mathrm{~m}$ & $44.48^{\circ}$ & $130 \mathrm{~m}$ \\
co-IFAM de Luk. & $0.70 \mathrm{~m}$ & $0.15^{\circ}$ & $106 \mathrm{~m}$ \\
co-IFAM de Gödel & $0.29 \mathrm{~m}$ & $0.55^{\circ}$ & $106 \mathrm{~m}$ \\
\hline
\end{tabular}

\begin{tabular}{lccc}
\hline & Erro $_{x, y}$ & Erro $_{\varphi t}$ & Dist \\
\hline Met. de Mamdani & $0.33 \mathrm{~m}$ & $0.40^{\circ}$ & $93 \mathrm{~m}$ \\
FAM de Kosko & $0.83 \mathrm{~m}$ & $0.15^{\circ}$ & $93 \mathrm{~m}$ \\
IFAM Lukasiewicz & $4.34 \mathrm{~m}$ & $43.37^{\circ}$ & $92 \mathrm{~m}$ \\
co-IFAM de Luk. & $0.32 \mathrm{~m}$ & $2.16^{\circ}$ & $92 \mathrm{~m}$ \\
co-IFAM de Gödel & $0.31 \mathrm{~m}$ & $0.04^{\circ}$ & $93 \mathrm{~m}$ \\
\hline
\end{tabular}

a) Posição inicial: $\left(20,30,-80^{\circ}, 60^{\circ}\right)$.

\begin{tabular}{lccc}
\hline & Erro $_{x, y}$ & Erro $_{\varphi \mathrm{t}}$ & Dist \\
\hline Met. de Mamdani & $0.87 \mathrm{~m}$ & $0.28^{\circ}$ & $109 \mathrm{~m}$ \\
FAM de Kosko & $0.32 \mathrm{~m}$ & $0.01^{\circ}$ & $108 \mathrm{~m}$ \\
IFAM Lukasiewicz & $10.04 \mathrm{~m}$ & $6.01^{\circ}$ & $138 \mathrm{~m}$ \\
co-IFAM de Luk. & $0.94 \mathrm{~m}$ & $0.46^{\circ}$ & $107 \mathrm{~m}$ \\
co-IFAM de Gödel & $0.53 \mathrm{~m}$ & $0.00^{\circ}$ & $105 \mathrm{~m}$ \\
\hline
\end{tabular}

b) Posição inicial: $\left(35,30,200^{\circ},-60^{\circ}\right)$.

\begin{tabular}{lccc}
\hline & Erro $_{\mathrm{x}, \mathrm{y}}$ & Erro $_{\varphi \mathrm{t}}$ & Dist \\
\hline Met. de Mamdani & $0.89 \mathrm{~m}$ & $0.76^{\circ}$ & $82 \mathrm{~m}$ \\
FAM de Kosko & $0.30 \mathrm{~m}$ & $0.10^{\circ}$ & $81 \mathrm{~m}$ \\
IFAM Lukasiewicz & $8.06 \mathrm{~m}$ & $27.10^{\circ}$ & $123 \mathrm{~m}$ \\
co-IFAM de Luk. & $0.14 \mathrm{~m}$ & $1.49^{\circ}$ & $81 \mathrm{~m}$ \\
co-IFAM de Gödel & $0.31 \mathrm{~m}$ & $1.01^{\circ}$ & $80 \mathrm{~m}$ \\
\hline
\end{tabular}

c) Posição inicial: $\left(45,30,-80^{\circ}, 30^{\circ}\right)$.

d) Posição inicial: $\left(55,30,160^{\circ}, 40^{\circ}\right)$.

\begin{tabular}{lccc}
\hline & Erro $_{x, y}$ & Erro $_{\varphi t}$ & Dist \\
\hline Met. de Mamdani & $0.07 \mathrm{~m}$ & $0.01^{\circ}$ & $109 \mathrm{~m}$ \\
FAM de Kosko & $0.39 \mathrm{~m}$ & $0.00^{\circ}$ & $109 \mathrm{~m}$ \\
IFAM Lukasiewicz & $8.13 \mathrm{~m}$ & $31.07^{\circ}$ & $146 \mathrm{~m}$ \\
co-IFAM de Luk. & $0.59 \mathrm{~m}$ & $1.51^{\circ}$ & $109 \mathrm{~m}$ \\
co-IFAM de Gödel & $0.47 \mathrm{~m}$ & $0.01^{\circ}$ & $109 \mathrm{~m}$ \\
\hline
\end{tabular}

e) Posição inicial: $\left(75,30,270^{\circ},-40^{\circ}\right)$.

\begin{tabular}{lccr}
\hline & Erro $_{\mathrm{x}, \mathrm{y}}$ & Erro $_{\varphi \mathrm{t}}$ & Dist \\
\hline Met. de Mamdani & $0.66 \mathrm{~m}$ & $0.83^{\circ}$ & $103 \mathrm{~m}$ \\
FAM de Kosko & $0.29 \mathrm{~m}$ & $0.48^{\circ}$ & $102 \mathrm{~m}$ \\
IFAM Lukasiewicz & $9.88 \mathrm{~m}$ & $7.36^{\circ}$ & $102 \mathrm{~m}$ \\
co-IFAM de Luk. & $0.75 \mathrm{~m}$ & $1.71^{\circ}$ & $103 \mathrm{~m}$ \\
co-IFAM de Gödel & $0.94 \mathrm{~m}$ & $0.08^{\circ}$ & $103 \mathrm{~m}$ \\
\hline
\end{tabular}

f) Posição inicial: $\left(80,20,10^{\circ}, 60^{\circ}\right)$.

A figura 7 apresenta pontos produzidos pelos controladores fuzzy para o problema BTT no plano cartesiano cujo eixo das abscissas e das ordenadas representam respectivamente o Erro $_{x, y}$ e a distância percorrida. Nessa figura, as letras correspondem as posições iniciais apresentadas na tabela 3 . Note que

o modelo de Mamdani apresentou um desempenho Pareto-ótimo para as posições iniciais a) e e). De fato, no gráfico da figura 7 , os pontos marcados com o símbolo " $\square$ " estão a esquerda de todos os demais pontos marcados com as letras "a" e "e". 
Tabela 4 - Regras de inferência do controlador fuzzy para manobrar a carreta.

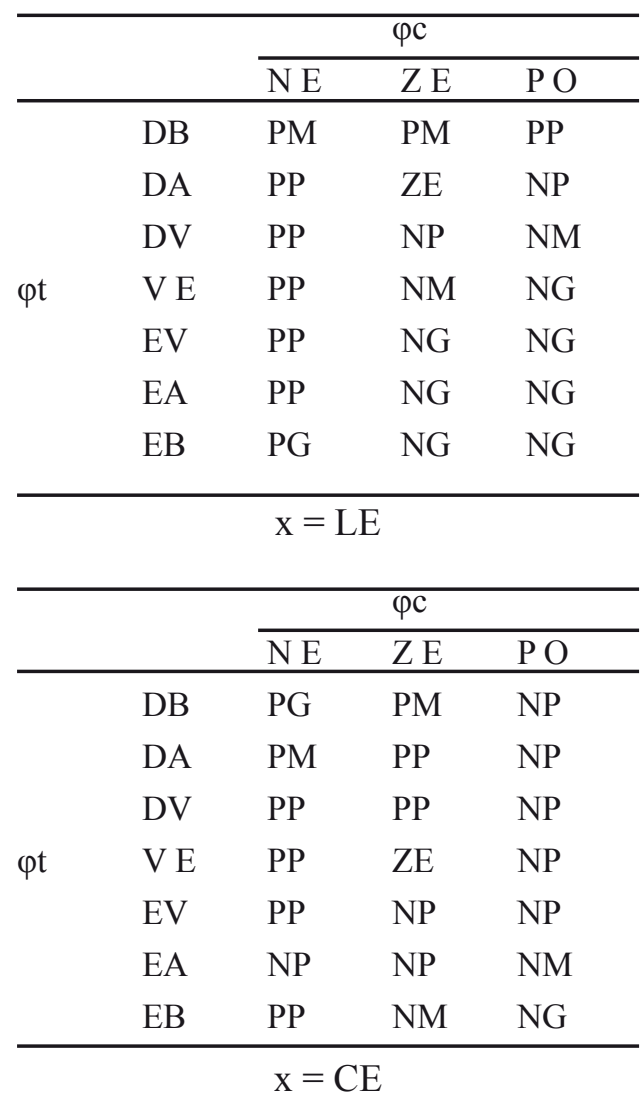

\begin{tabular}{lllll}
\hline & & \multicolumn{3}{l}{$\varphi \mathrm{c}$} \\
\cline { 3 - 5 } & & $\mathrm{N} \mathrm{E}$ & $\mathrm{Z} \mathrm{E}$ & $\mathrm{P} \mathrm{O}$ \\
\hline \multirow{4}{*}{$\varphi \mathrm{DB}$} & $\mathrm{PM}$ & $\mathrm{PM}$ & $\mathrm{NP}$ \\
& $\mathrm{DA}$ & $\mathrm{ZE}$ & $\mathrm{PP}$ & $\mathrm{NP}$ \\
& $\mathrm{DV}$ & $\mathrm{PP}$ & $\mathrm{NP}$ & $\mathrm{NM}$ \\
& $\mathrm{V} \mathrm{E}$ & $\mathrm{PP}$ & $\mathrm{NP}$ & $\mathrm{NM}$ \\
& $\mathrm{EV}$ & $\mathrm{PP}$ & $\mathrm{NM}$ & $\mathrm{NM}$ \\
& $\mathrm{EA}$ & $\mathrm{PP}$ & $\mathrm{NG}$ & $\mathrm{NG}$ \\
& $\mathrm{EB}$ & $\mathrm{PP}$ & $\mathrm{NG}$ & $\mathrm{NG}$ \\
\hline
\end{tabular}

$$
\mathrm{x}=\mathrm{LC}
$$

\begin{tabular}{llll}
\hline & \multicolumn{3}{c}{$\varphi \mathrm{c}$} \\
\cline { 2 - 4 } & $\mathrm{NE}$ & $\mathrm{ZE}$ & $\mathrm{PO}$ \\
\hline $\mathrm{DB}$ & $\mathrm{PG}$ & $\mathrm{PG}$ & $\mathrm{NP}$
\end{tabular}

DA PG PG NP

DV PM PM NP

$\varphi t \quad \mathrm{VE} \quad \mathrm{PM} \quad \mathrm{PP} \quad \mathrm{NP}$

EV PM PP NP

\begin{tabular}{llll} 
EA & PP & NP & ZE \\
EB & PP & NM & NM \\
\hline \multicolumn{3}{c}{$\mathrm{x}=\mathrm{DC}$}
\end{tabular}

\begin{tabular}{cllll}
\hline & & \multicolumn{3}{c}{$\varphi \mathrm{c}$} \\
\cline { 3 - 5 } & & $\mathrm{NE}$ & $\mathrm{Z} \mathrm{E}$ & $\mathrm{P} \mathrm{O}$ \\
\hline \multirow{4}{*}{ DB } & PG & PG & NG \\
& DA & PG & PG & NP \\
& DV & PG & PG & NP \\
& V E & PG & PM & NP \\
& EV & PM & PP & NP \\
& EA & PP & ZE & NP \\
& EB & PP & NM & NM \\
\hline \multicolumn{4}{c}{$\mathrm{x}=$ DI }
\end{tabular}

De um modo similar, concluímos que o modelo de Kosko apresentou um desempenho Pareto-ótimo para o ponto inicial f). Para os pontos iniciais b) e d), os controladores baseados nas co-IFAMs de Gödel e Lukasiewicz apresentaram desempenho Pareto-ótimo. Finalmente, a FAM de Kosko e a coIFAM de Gödel apresentaram desempenho Paretoótimo para o ponto inicial c). A tabela 5 apresenta um resumo dos controladores que apresentaram desempenho Pareto-ótimo para cada ponto inicial.
Tabela 5 - Resumo dos controladores que apresentaram desempenho Pareto-ótimo.

\begin{tabular}{lc}
\hline Ponto Inicial & Controlador \\
\hline a) & Método de Mamdani \\
b) & co-IFAMs de Gödel e Lukasiewicz \\
c) & FAM de Kosko e co-IFAM de Gödel \\
d) & co-IFAMs de Gödel e Lukasiewicz \\
e) & Método de Mamdani \\
f) & FAM de Kosko \\
\hline
\end{tabular}


Figura 6. Trajetórias produzidas pelos controladores fuzzy baseados em FAMs. Os símbolos “ $\nabla$ " (vermelho) e “ $\square$ " (azul) representam a trajetória da cabine e da carreta, respectivamente.
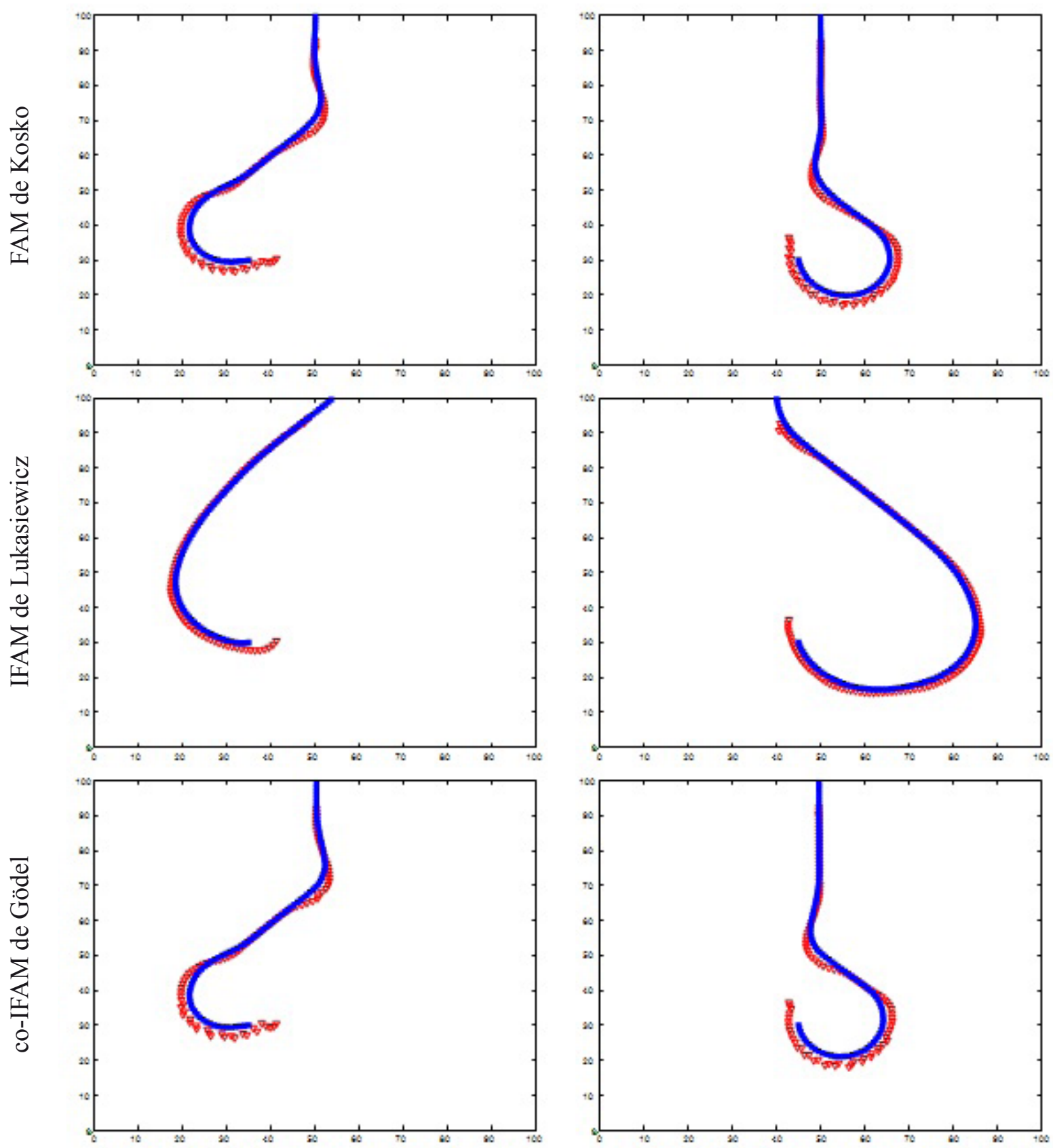

a) Posição inicial: $\left(35,30,200^{\circ},-60^{\circ}\right)$

b) Posição inicial: $\left(45,30,-80^{\circ}, 30^{\circ}\right)$ 
Figura 7. Desempenho dos controladores fuzzy para o problema BTT.

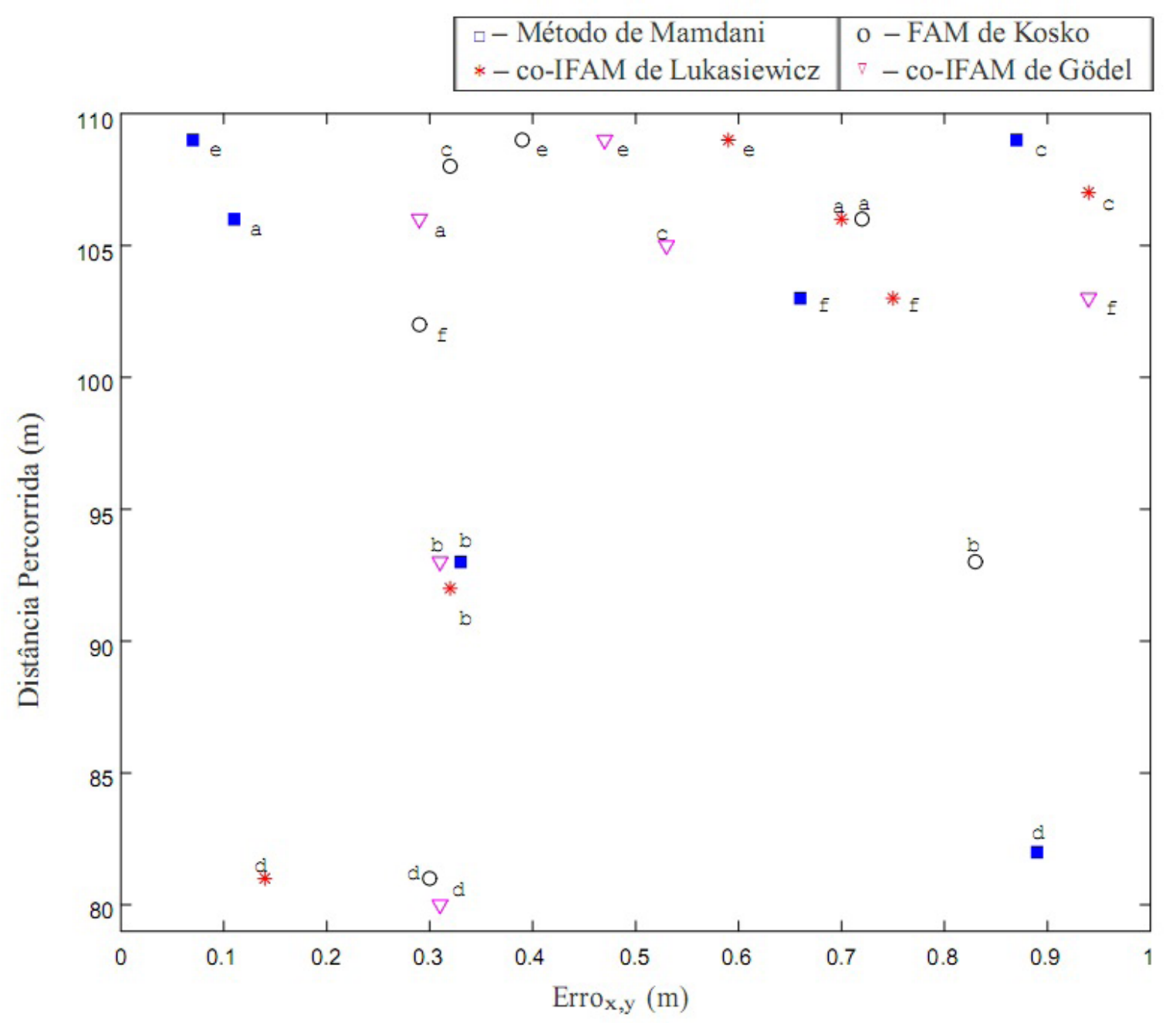

\section{Conclusões}

Primeiramente, gostaríamos de lembrar que a IFAM de Lukasiewicz apresentou excelentes resultados em problemas de reconstrução de imagens (SUSSNER; VALLE, 2006a; VALLE, 2007). Esse modelo, entretanto, apresentou um comportamento pouco satisfatório - trajetórias longas e erros de estacionamento grandes - tanto no problema BT quanto no BTT. Todavia, acreditamos que resultados melhores podem ser obtidos modificando os conjuntos e as regras fuzzy usadas nesse problema.

As IFAMs duais, por outro lado, apresentaram um desempenho semelhante aos modelos tradicionais de
Kosko e de Mamdani. De fato, no problema BTT, as co-IFAMs apresen taram desempenho Pareto-ótimo para alguns pontos iniciais. Essa observação revela que as IFAMs duais representam uma alternativa atraente aos modelos tradicionais. No futuro, pretendemos efetuar uma investigação mais extensa desses modelos em problemas de controle. Em particular, iremos avaliar o desempenho de outras IFAMs duais, como a co-IFAM baseada na coimplicação de Göguen, em problemas de guiagem autônoma.

\section{Agradecimentos}

Este trabalho contou com o apoio daFUNDAÇÃO 
ARAUCÁRIA de Apoio ao Desenvolvimento Científico e Tecnológico do Paraná.

\section{Referências}

CHEN, S.-M.; YEH, M.-S.; HSIAO, P.-Y. A comparison of similarity measures of fuzzy values. Fuzzy Sets and Systems, Amsterdam, v. 72, n. 1, p. 79-89, maio 1995.

CHUNG, F.; LEE, T. On fuzzy associative memory with multiple-rule storage capacity. IEEE Transactions on Fuzzy Systems, New York, v. 4, n. 3, p. 375-384, 1996.

COELLO, C. A. C.; LAMONT, G. B.; VELDHUIZEN, D. A. V. Evolutionary algorithms for solving multiobjective problems. New York: Springer, 2007. (Genetic and Evolutionary Computation Series).

DE BAETS, B. Fuzzy morphology: a logical approach. In: AYYUB, B. M.; GUPTA, M. M. (Ed.). Uncertainty analysis in engineering and science: fuzzy logic, statistics, and neural network approach. Norwell: Kluwer Academic Publishers, 1997. p. 53-67.

DUBOIS, D.; PRADE, H. Fuzzy sets and systems: theory and applications. New York: Academic Press, 1980.

IBRAHIM, A. M. Fuzzy logica for embedded systems applications. Burlington: Elsevier Science, 2004.

JANTZEN, J. Foundations of fuzzy control. New York: John Wiley \& Sons, 2007.

KLIR, G. J.; YUAN, B. Fuzzy sets and fuzzy logic: theory and applications. Upper Saddle River: Prentice Hall, 1995.

KOGA, T.; HORIO, K.; YAMAKAWA, T. The selforganizing relationship (SOR) network employing fuzzy inference based heuristic evaluation. Neural Networks, New York, v. 19, n. 6, p. 799-811, 2006.

KONG, S.-G.; KOSKO, B. Adaptive fuzzy systems for backing up a truck-and-trailer. IEEE Transactions on Neural Networks, New York, v. 3, n. 2, p. 211-223, 1992.

KOSKO, B. Neural networks and fuzzy systems: a dynamical systems approach to machine intelligence. Englewood Cliffs: Prentice Hall, 1992.

LEE, C. Fuzzy logic in control systems: fuzzy logic controller. i. Systems, Man and Cybernetics, IEEE Transactions on, New York, v. 20, n. 2, p. 404-418, Mar./ Apr. 1990a.

Fuzzy logic in control systems: fuzzy logic controller. ii. Systems, Man and Cybernetics, IEEE Transactions on, New York, v. 20, n. 2, p. 419-435, Mar./ Apr. 1990b.
NGUYEN, D.; WIDROW, B. Truck backer-upper: an example of self-learning in neural networks. In: IEEE INTERNATIONAL JOINT CONFERENCE ON NEURAL NETWORKS, 1989, Washington. Proceedings... Washington: [s.n.], 1989. p. 357-363.

OLIVEIRA, R. M. Caracterização e continuidade das memórias associativas morfológicas fuzzy baseadas em uninormas. 2010. Dissertação (Mestrado em Matemática Aplicada e Computacional) - Universidade Estadual de Londrina, Londrina.

PAPPIS, C. P.; KARACAPILIDIS, N. I. A comparative assessment of measures of similarity of fuzzy values. Fuzzy Sets and Systems, Amsterdam, v. 56, n. 2, p. 171174, jun. 1993.

PEDRYCZ, W.; GOMIDE, F. Fuzzy systems engineering: toward human-centric computing. New York: WileyIEEE Press, 2007.

PEDRYCZ, W.; SKOWRON, A.; KREINOVICH, V. (Ed.). Handbook of granular computing. New York: John Wiley and Sons, 2008.

PERFILIEVA, I.; LEHMKE, S. Correct models of fuzzy ifthen rules are continuous. Fuzzy Sets and Systems, Amsterdam, v. 157, n. 24, p. 3188-3197, 2006.

RIID, A.; RÜSTERN, E. Fuzzy logic in control: truck backer-upper problem revisited. In: IEEE INTERNATIONAL CONFERENCE ON FUZZY SYSTEMS, 10., 2001, Melbourne. Proceedings... Melbourne: [s.n.], 2001. p. 513-516. v. 1.

STADLER, W. Fundamentals of multicriteria optimization. In: _. Multicriteria optimization in engineering and the sciences. New York: Plenum Press, 1988. p. 1-25.

SUSSNER, P.; MIYASAKI, R.; VALLE, M. E. An introduction to parameterized IFAM models with applications in pre- diction. In: IFSA WORLD CONGRESS AND EUSFLAT CONFERENCE, 2009, Lisbon. Proceedings... Lisbon: [s.n.], 2009. p. 247-252.

SUSSNER, P.; VALLE, M. E. Implicative fuzzy associative memories. IEEE Transactions on Fuzzy Systems, New York, v. 14, n. 6, p. 793-807, 2006 a.

Recall of patterns using morphological and certain fuzzy morphological associative memories. In: IEEE WORLD CONFERENCE ONCOMPUTATIONAL INTELLIGENCE, 2006, Vancouver. Proceedings... Vancouver: [s.n.], 2006b. p. 209-216.

. Morphological and certain fuzzy morphological associative memories for classification and prediction. In: KABURLASOS, V.; RITTER, G. (Ed.). Computational intelligence based on lattice theory. Heidelberg: Springer- 
Verlag, 2007. p. 149-172.

TANAKA, K.; KOSAKI, T. Design of a stable fuzzy controller for an articulated vehicle. IEEE Transaction on Systems, Man, and Cybernetics, Part B: Cybernetics, New York, v. 27, n. 3, p. 552-558, Jun. 1997.

VALLE, M. E. Fundamentos e aplicações de memórias associativas morfológicas nebulosas. 2007. Tese (Doutorado em Matemática Aplicada) - Universidade Estadual de Campinas, Campinas.

Sparsely connected autoassociative fuzzy implicative memories for the reconstruction of large grayscale images. In: IFSA WORLD CONGRESS AND EUSFLAT CONFERENCE, 2009, Lisbon. Proceedings... Lisbon: [s.n.], 2009. p. 247-252.

Sparsely connected autoassociative fuzzy implicative memories and their application for reconstruction of large gray-scale images. Neurocomputing, Netherlands, v. 74, n. 1-3, p. 343-353, dez. 2010.

VALLE, M. E.; SUSSNER, P. Fuzzy morphological associative memories based on uninorms. In: IEEE WORLD CONFERENCE ON COMPUTATIONAL INTELLIGENCE, 2008, Hong Kong. Proceedings... Hong Kong: [s.n.], 2008. p. 1582-1589.

WANG, J.; ZHANG, Y. Backing up truck control automatically based on los. In: INTERNATIONAL CONFERENCE ON HYBRID INTELLIGENT SYSTEMS, 9., 2009, Washington. Proceedings... Washington: [s.n.], 2009. p. 108-111. v. 2.

YANG, X.; YUAN, J.; YU, F. Backing up a truck and trailer using variable universe based fuzzy controller. In: IEEE INTERNATIONAL CONFERENCE ON MECHATRONICS AND AUTOMATION, 2006, Luoyang. Proceedings... Luoyang: [s.n.], 2006. p. 734739.

Recebido em 18 Agosto 2010-Received on August 18, 2010.

Aceito em 19 Maio, 2011 - Accepted on May 19, 2011. 
Freitas, G. A. L.; Valle, M. E. 